\title{
In vitro enhancement and functional characterization of neurite outgrowth by undifferentiated adipose-derived stem cells
}

\author{
VESNA BUCAN $^{1}{ }^{,}$MALTE FLIESS $^{1}$, REINHILD SCHNABEL $^{1}$, CLAAS-TIDO PECK $^{1}$, DESIREE VASLAITIS ${ }^{1}$, \\ ANGELA FÜLBIER $^{1}$, KERSTIN REIMERS ${ }^{1}$, SARAH STRAUSS $^{1}$, PETER M. VOGT $^{1}$ and CHRISTINE RADTKE ${ }^{1,2}$ \\ ${ }^{1}$ Department of Plastic, Aesthetic, Hand and Reconstructive Surgery, Hannover Medical School, D-30625 Hannover, Germany; \\ ${ }^{2}$ Department of Plastic and Reconstructive Surgery, Medical University of Vienna, A-1090 Vienna, Austria
}

Received February 22, 2018; Accepted October 22, 2018

DOI: $10.3892 /$ ijmm.2018.3979

\begin{abstract}
Adipose-derived stem cells (ASCs) can easily be obtained and expanded in vitro for use in autologous cell therapy. Via their production of cytokines and neurotrophic factors, transplanted ASCs provide neuroprotection, neovascularization and induction of axonal sprouting. However, the influencing mechanism of undifferentiated ASCs on nerve regeneration is currently only partially understood. In the present study, undifferentiated ASCs and cutaneous primary afferent dorsal root ganglion (DRG) neurons were co-cultured in order to investigate their interaction. ASCs were isolated from adult rat fat tissue. The presence of characteristic stem cell markers was determined by flow cytometry in three subsequent passages. Adipogenic, osteogenic, chondrogenic and glial differentiation was performed in order to evaluate their differentiation capacity. A direct co-culture system with DRG cells was established to determine the effect of undifferentiated pluripotent ASCs on neurite elongation. Neurite outgrowth, length and number was examined in the co-culture and compared with single-culture cells and cells stimulated with nerve growth factor (NGF). In ASC cultures, NGF expression was assessed by ELISA. The present results demonstrated that the specific mesenchymal stem cell surface markers CD44, CD73 and CD90 were detected in all three subsequent passages of the isolated ASCs. In accordance, ASC differentiation into adipogenic, osteogenic, chondrogenic and Schwann cell phenotype was conducted successfully. Neurite outgrowth of DRG neurons was enhanced following co-culture with ASCs, resulting in increased neurite length after $24 \mathrm{~h}$ of cultivation. Furthermore, neurite outgrowth of DRG neurons was directed towards the undifferentiated ASC and direct cell-to-cell contact was observed. In summary, the results of the present study revealed an interaction between
\end{abstract}

Correspondence to: Dr Vesna Bucan, Department of Plastic, Aesthetic, Hand and Reconstructive Surgery, Hannover Medical School, 21 Feodor-Lynen Street, D-30625 Hannover, Germany E-mail: bucan.vesna@mh-hannover.de

Key words: adipose-derived stem cell, nerve regeneration, stem cell and neuron co-culture, growth factor, cell-to-cell contact the two cell types with guidance of neurite growth towards the undifferentiated ASC. These findings suggest that the use of undifferentiated ASC optimizing tissue-engineered constructs may be promising for peripheral nerve repair.

\section{Introduction}

Peripheral nerve injury can be caused by accidents, idiopathic damages, iatrogenic injuries or systemic diseases and is typically characterized by loss of sensory and motoric functions downstream of the defect. Approximately 300,000 cases of peripheral nerve injury are reported every year in Europe alone. However, unlike the central nervous system, the peripheral nervous system is characterized by a much higher intrinsic potential to regenerate (1). The current clinical gold standard for the treatment of peripheral nerve injury is the end-to-end suturing of nerves. In cases where tension-free suture is not possible due to substantial loss of nerve tissue, autologous nerve grafts are used (2). This, however, shifts the problem of nerve defect to the donor site. Furthermore, it has been demonstrated that, in peripheral nerve surgery, microsurgery of the severed nerve alone fails to address extensive cell death in the dorsal root ganglia (3). As a result, different research approaches to enhance regeneration of peripheral nerve injuries are being explored.

One such potential approach to enhance nerve regeneration is to administer exogenous growth factors, either directly or entrapped into biodegradable polymeric nano- and microparticles for localized delivery (including silicone, PLGA, PLA or polyphosphoesters of ethylene terephthalate), which may promote axonal regeneration (4). However, the success in terms of functional nerve regeneration of the described systems and related treatments has been limited. One reason for this failure might be the limitations of the delivery systems, such as inadequate release kinetics, loss of bioactivity, single (rather than multiple) factor delivery and lack of cellular ingrowth. Cell-based delivery systems could be an alternative, since the cells can be engineered to overexpress specific growth factors and deliver these agents locally (2).

Another potential approach that is being investigated is to transplant other cell types into the nerve grafts to aid the process of regeneration, including Schwann cells (SCs) (5), olfactory ensheathing cells (OECs) (6), bone marrow-derived stem 
cells (BMSCs) (7), adipose-derived stem cells (ASCs) $(8,9)$ and induced pluripotent stem cells (iPSCs) (10). Stem cells have the potential to increase the number of SCs and prolong their ability to support regeneration. Furthermore, stem cells can promote peripheral nerve regeneration by their ability to differentiate into a SC phenotype, secrete neurotrophic factors, essential for regeneration, and their potential for myelin formation (11). Though SCs are the most fundamental cell type for peripheral nerve regeneration, their clinical application is limited due to the inability to isolate a sufficient amount of these cells in a short period of time (12). Tohill and Terenghi (7), reported that, under appropriate conditions, BMSCs can differentiate into non-mesodermal lineages, such as neurons, astrocytes and SC-like cells (7). Multiple studies have demonstrated that iPSCs have a pro-regenerative effect in small animal models of central and peripheral nervous system injury (13-16).

The use of ASCs, in specific, may have practical and clinical advantages, since they are characterized by a high proliferation rate and can be derived by liposuction from subcutaneous fat tissue in large quantities with limited donor site morbidity and discomfort (17). Approximately 400,000 liposuction procedures are conducted in the US every year. Each procedure yields $100-3,000 \mathrm{ml}$ of lipoaspirate tissue (18), which is a heterogeneous mixture of cells with a high number of ASCs (19). Several protocols have been established to induce neuronal differentiation of undifferentiated ASCs, such as treatment with epidermal growth factor and basic fibroblast growth factor (bFGF) in a neural progenitor basal medium resulting in floating neurosphere induction (20); additionally, differentiated cells have been used in cell therapeutic approaches (3,13-26).

Although ASCs have the clear ability to myelinate dorsal root ganglion (DRG) neurons in co-culture $(27,28)$, long-term survival of the transplanted cells has been questioned in a study directly addressing this problem (29). Thus it has been hypothesized that the beneficial effect of ASCs in neuroregeneration depends to a large extent on secretion of neurotrophic factors, reduction of inflammation and neuronal loss (30). For instance, the incorporation of differentiated ASCs into dorsal root ganglia significantly increased anti-apoptotic Bcl-2 mRNA expression in DRG neurons (23). Angiogenesis is also an important factor (31). Nevertheless, the intrinsic properties of cells in contact with neurons need to be explored in further detail as a prerequisite for optimal application of ASCs to enhance and support peripheral nerve regeneration.

Different strategies have been developed to exploit the therapeutic benefit of ASCs applied to neural damage. While lipoaspirated adipose tissue filled in a segment of epigastric vein impaired functional recovery of a $10 \mathrm{~mm}$ gap in a rat sciatic nerve, processing of the adipose tissue to obtain either the stromal vascular fraction or even ASCs appears important in the context of nerve regeneration (22).

Branching of neurons at the site of injury is known to be a dynamic process, generally decreasing over time, as branches that fail to establish functional contact with the periphery are believed to be eliminated. In the present study, the immediate effect of undifferentiated ASC-DRG neuron interaction was examined by a detailed analysis of DRG neurite outgrowth over 24 and $48 \mathrm{~h}$ in comparison to single-culture cells and cells stimulated with nerve growth factor (NGF).

\section{Materials and methods}

ASC harvest and cell culture. All animals were treated according to the legal and ethical requirements of the German Animal Welfare Act and were approved by the Animal Ethics Committee of the Hannover Medical School Central Animal Laboratory (approval no. 2014/52). Adipose tissue was obtained from inguinal fat depots of adult male Lewis rats, 8 weeks old, weighing 350-400 g ( $\mathrm{n}=12)$ supplied by Charles River Laboratories (Sulzfeld, Germany). The fat pads were carefully dissected from the rats under inhalation isoflurane anesthesia, subsequently the animals were sacrificed. After rinsing with Hank's balanced salt solution (HBSS; PAA Laboratories; GE Healthcare GmbH, Solingen, Germany) and mincing, the fat tissue was digested with collagenase type I, CLS I ( $2 \mathrm{mg} / \mathrm{ml}$; Biochrom GmbH, Berlin, Germany) for $60 \mathrm{~min}$ at $37^{\circ} \mathrm{C}$ under shaking. Following centrifugation for $10 \mathrm{~min}$ at $620 \mathrm{x} \mathrm{g}$, at room temperature, the cell pellet was resuspended in ASC culture medium: DMEM/F12 (Biochrom $\mathrm{GmbH}$ ) with $100 \mathrm{U} / \mathrm{ml}$ penicillin, $100 \mathrm{mg} / \mathrm{m}$ streptomycin, $0.2 \mathrm{mM}$ L-ascorbic acid-2-phosphate (Sigma-Aldrich; Merck KGaA, Darmstadt, Germany), and $10 \%$ fetal bovine serum (FBS; Biochrom $\mathrm{GmbH}$ ). Cells were plated on two $150 \mathrm{~cm}^{2}$ cell culture flasks under standard conditions at $37^{\circ} \mathrm{C}, 100 \%$ atmosphere humidity and $5 \% \mathrm{CO}_{2}$.

Phenotypic characterization via surface marker expression and flow cytometry. Cultured cells (passage 1-3, $\mathrm{n}=6$ per passage) were examined for ASC-specific surface markers using flow cytometry. The following anti-rat antibodies conjugated to fluorochromes or unconjugated were used: CD11b/c PerCP-eFluor 710 (cat. no. MA1-81606; eBioscience; Thermo Fisher Scientific, Inc., Waltham, MA, USA), CD44H-fluorescein isothiocyanate (FITC; cat. no. 550974) and CD105-FITC (cat. no. 562762; BD Biosciences, San Jose, CA, USA), CD45-FITC (cat. no. 202205) and CD90-PE/CY7 (cat. no. 328123; BioLegend, Inc., Fell, Germany), CD34 (cat. no. sc-7324; Santa Cruz Biotechnology, Inc., Dallas, TX, USA) and CD73 (cat. no. 551123; BD Pharmingen; BD Biosciences). For immunolabelling, cells were detached from culture flasks with $0.2 \%$ EDTA solution and washed with PBS containing 10\% FBS. The cells were blocked with $1 \% \mathrm{BSA}$ in PBS for $30 \mathrm{~min}$ at $4^{\circ} \mathrm{C}$, centrifuged for $5 \mathrm{~min}$ at $200 \mathrm{x} \mathrm{g}$, at room temperature, resuspended in PBS and incubated with the primary antibodies (final dilution 1:10) for $50 \mathrm{~min}$ at $4^{\circ} \mathrm{C}$. After washing with PBS, the probes with unconjugated primary antibodies were incubated with 1:10 diluted fluorochrome-labeled secondary antibodies bovine anti-goat IgG-PerCP-Cy5.5 or goat-anti-mouse-IgG-PE (both Santa Cruz Biotechnology, Inc.) for $45 \mathrm{~min}$ at $4^{\circ} \mathrm{C}$, washed and analyzed. The probes with conjugated antibodies were analyzed directly after washing with PBS. A FC500 flow cytometer with CXP-software v2.2 (Beckman Coulter, Inc., Krefeld, Germany) was used for evaluation. The means and standard deviations were calculated and statistical significance was evaluated by analysis of variance (ANOVA) and Bonferroni's post hoc test.

Induction of adipogenic, osteogenic, chondrogenic and SC differentiation. In preparation of adipogenic and osteogenic 
differentiation, ASCs in passage 2 were plated at a cell density of $3 \times 10^{4}$ cells $/ \mathrm{cm}^{2}$ into $40 \mathrm{~mm}$ dishes and allowed to adhere for $20 \mathrm{~h}$. To induce adipogenic differentiation, the samples were cultured in standard ASC medium supplemented with $1 \mu \mathrm{M}$ dexamethasone, $0.5 \mathrm{mM}$ 1-methyl-3-isobutylxanthine, $1 \mathrm{ng} / \mathrm{ml}$ insulin and $100 \mu \mathrm{M}$ indomethacin (all from Sigma-Aldrich; Merck KGaA) for 3 weeks. To stimulate osteogenic differentiation, $100 \mathrm{nM}$ dexamethasone and $3 \mathrm{mM}$ $\beta$-glycerophosphate (Sigma-Aldrich; Merck KGaA) were added to standard medium over a period of 4 weeks. After fixation with $4 \%(\mathrm{w} / \mathrm{v})$ phosphate buffered paraformaldehyde (PFA; Carl Roth Gmbh \& Co. Kg, Karlsruhe, Germany), for $15 \mathrm{~min}$ at room temperature, differentiation to fat cells was visualized by staining lipid droplets with Oil Red O (Serva, Mannheim, Germany), for $10 \mathrm{~min}$ at room temperature, while osteogenic cells were detected by staining of calcium depositions with Alizarin red (Sigma-Aldrich; Merck KGaA) for $1 \mathrm{~h}$ at room temperature.

To induce chondrogenic differentiation, $3 \times 10^{6}$ cells were incubated as pellets in an upright centrifugation tube at $37^{\circ} \mathrm{C}, 5 \% \mathrm{CO}_{2}$ and $100 \%$ humidity for three weeks. The differentiated cell pellets were washed with PBS and fixed with $4 \%$ paraformaldehyde in PBS, dehydrated, embedded in paraffin, sectioned and stained with Alcian blue.

Differentiation to SCs was induced by supplementing standard medium with $1 \%$ ITS + (BD Biosciences), $1 \mathrm{mM}$ sodium pyruvate (Biochrom $\mathrm{GmbH}$ ), $40 \mu \mathrm{g} / \mathrm{ml}$ proline, $40 \mathrm{ng} / \mathrm{ml}$ dexamethasone (both from Sigma-Aldrich; Merck KGaA), 10 ng/mg TGF $\beta 1$ (PeproTech, Ltd., Rocky Hill, NJ, USA). Successful differentiation was evaluated by immunocytochemistry, as described by Kingham et al (32). For this purpose differentiated cells were trypsinised and replated on $\mu$-slides (ibidi $\mathrm{GmbH}$, Martinsried, Germany) for 2 days. Subsequently, cells were fixed with $4 \%$ PFA for $15 \mathrm{~min}$ at room temperature and immunostained based on a standard protocol using the primary antibody rabbit anti-S100 (1:500; Dako; Agilent Technologies GmbH, Waldbronn, Germany) and the secondary antibody goat-anti-rabbit Alexa Fluor 555 (1:400; Invitrogen; Thermo Fisher Scientific, Inc.).

Cell size analysis and proliferation assay. Cultured cells (passages 1-3, $n=4$ per passage) were analyzed morphometrically, after confluence of $50-70 \%$ was reached. A total of five random digital images $(2,080 \times 1,544$ pixels, 1.15 pixels $/ \mu \mathrm{m})$ at magnification, $\mathrm{x} 4$ were captured with an Olympus CKX41 microscope with Color View Soft imaging system (Olympus Corporation, Tokyo, Japan). Within these images, the surface area occupied by 200 cells was measured using the public domain Java-based image processing and analysis program ImageJ_v1.15g (National Institutes of Health, Bethesda, MD, USA).

At $80-90 \%$ confluence, cells were detached with trypsin-EDTA solution (0.25\%/0.02\%; Biochrom $\mathrm{GmbH})$, washed, seeded in 6-well plates at 3,000 cells $/ \mathrm{cm}^{2}$ and incubated under standard conditions. After $24 \mathrm{~h}$, the cells of three wells were detached and counted using a Neubauer haemocytometer. The cells in the remaining three wells were detached and counted following $48 \mathrm{~h}$ of incubation. Dead cells were identified by trypan blue and subtracted from the cell count. The proliferation factor was calculated as the quotient of the cell count at $48 \mathrm{~h}$ and the cell count at $24 \mathrm{~h}$ for each passage. Means and standard deviations were calculated and statistical significance was evaluated by ANOVA followed by Bonferroni's post hoc test.

$D R G$ harvest and culture. DRG neurons were obtained from 10-week old male Wistar rats weighing 400-500 g $(n=8)$ supplied by Charles River Laboratories (Wilmington, MA, USA). Animals were sacrificed under isoflurane anesthesia, ganglia were extracted, washed and incubated in HBSS containing $1.7 \mathrm{mg} / \mathrm{ml}$ collagenase A (Roche Diagnostics $\mathrm{GmbH}$, Mannheim, Germany). The digested ganglia were gently dissociated in DMEM/F12 + 6\% D-glucose (Merck $\mathrm{KGaA}$ ), centrifuged and washed. Afterwards, a previously prepared 20\% BSA solution in DMEM/F12 was overlaid with the resuspended cells and centrifuged. After the liquid layers containing debris were removed and the neurons at the base were resuspended in a modified Bottenstein and Sato medium [DRG medium: DMEM/F12 + 6\% D-glucose supplemented with $100 \mu \mathrm{g} / \mathrm{ml} \mathrm{BSA}, 100 \mu \mathrm{g} / \mathrm{ml}$ transferrin, $100 \mu \mathrm{M}$ putrescine, $30 \mathrm{nM}$ sodium selenite, $20 \mathrm{nM}$ progesterone, $10 \mathrm{nM}$ insulin (all purchased from Sigma-Aldrich; Merck KGaA), $100 \mathrm{U} / \mathrm{ml}$ penicillin and $100 \mathrm{mg} / \mathrm{ml}$ streptomycin (PAA)], the neurons were counted and plated onto laminin-coated $12 \mathrm{~mm}$ glass coverslips in 24-well plates at 1,000 neurons/coverslip.

DRG neuron and ASC co-culture. The DRG neurons were cultured with ASCs of passage 2. The cultures were maintained in an incubator at $37^{\circ} \mathrm{C}$ with humidified atmosphere and $5 \% \mathrm{CO}_{2}$. As a negative control, neurons were cultured separately without ASCs in normal DRG medium and, as a positive control, neurons were cultured without ASCs in DRG medium supplemented with $10 \mathrm{ng} / \mathrm{ml}$ NGF (Sigma-Aldrich; Merck $\mathrm{KGaA}$ ). After 24 and $48 \mathrm{~h}$, the cells were fixed with $4 \%$ PFA for $15 \mathrm{~min}$, at room temperature. For immunofluorescence, anti-tubulin $\beta$ III (MMS-435P; Covance, Inc., Princeton, NJ, USA) was used as primary antibody and Alexa Fluor 555 anti-rabbit IgG (cat. no. A32732; Molecular Probes; Thermo Fisher Scientific, Inc.) as secondary antibody. The sections were counterstained with DAPI (Sigma-Aldrich; Merck KGaA). Phase contrast images $(2,080 \times 1,544$ pixels, 2.9 pixels $/ \mu \mathrm{m})$ of all neurons were taken at x10 objective magnification using the CKX41 imaging system (Olympus Corporation). Neurons were analyzed morphometrically using ImageJ software, with the number and length of outgrowing neurites were measured. Means and standard deviations were calculated and tested for statistical significance by ANOVA and Bonferroni's post hoc test (soma diameter), or Kruskal-Wallis-ANOVA followed by Bonferroni corrected Mann-Whitney U-Test (number and length of neurites).

Statistical analysis. Data are presented as means + standard deviation. Differences between groups were evaluated by ANOVA and Bonferroni's post hoc test (soma diameter), or Kruskal-Wallis-ANOVA followed by Bonferroni corrected Mann-Whitney U-Test (number and length of neurites). Statistics based on the IBM SPSS v.20. (IBM, Corp., Armonk, NY, USA). $\mathrm{P}<0.05$ was considered to indicate a statistically significant difference. 

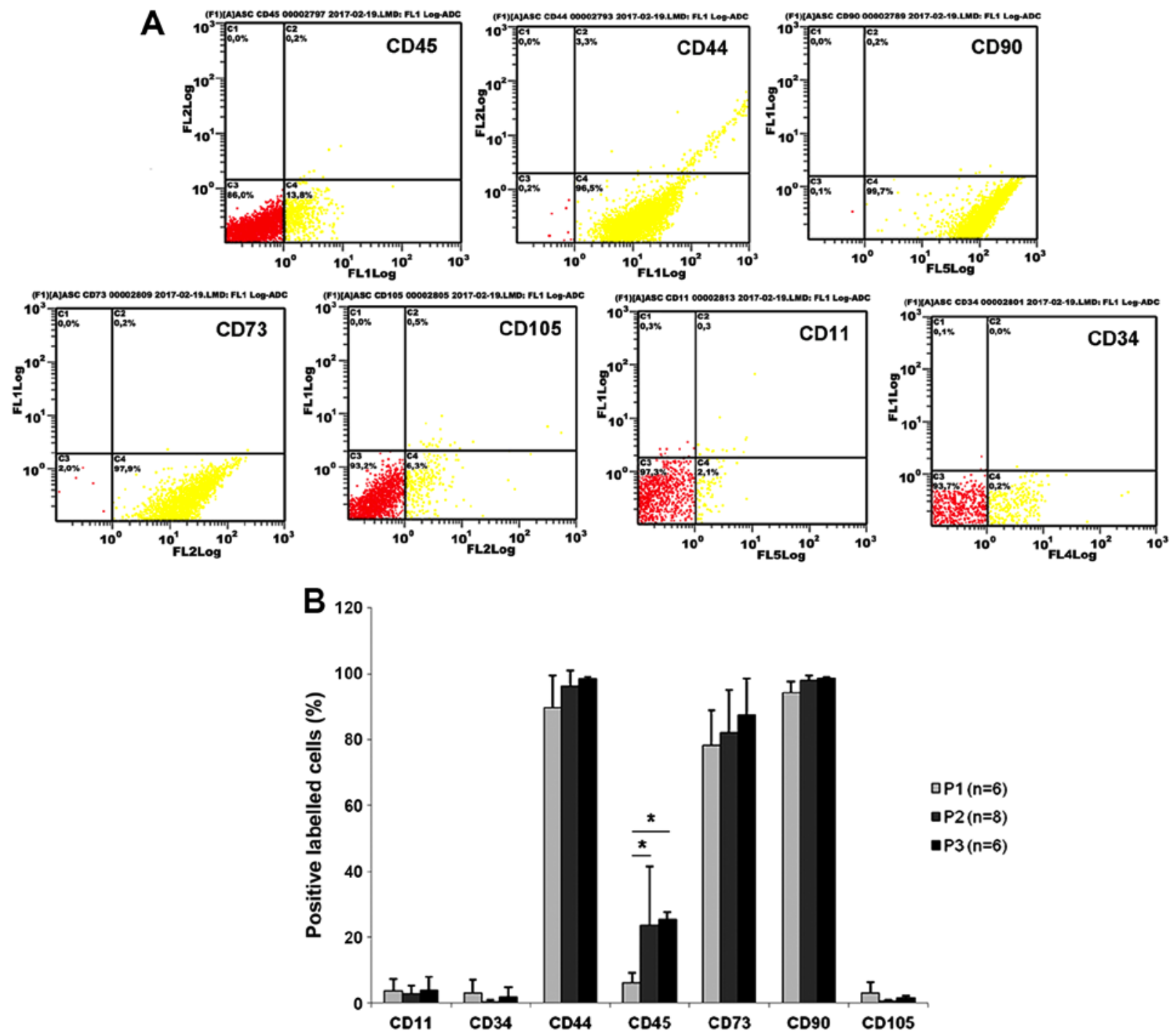

Figure 1. Phenotypic characterization of ASCs by flow cytometry and changes with passaging. (A) Representative plots from flow cytometry analyses. (B) Quantification of positive cells, \% over total. At all three passages, the majority of cells expressed the specific mesenchymal stem cell surface markers CD44, CD73 and CD90, whereas $<7 \%$ of the cells were positive for CD11, CD105 and CD34. The \% of CD45 was 6.15 $\pm 2.99 \%$ at passage 1 and increased to $42.36 \pm 2.18 \%$ at passage 3 . Data are presented as means + standard deviation. ${ }^{*} \mathrm{P}<0.05$ with comparisons indicated by lines. ASCs, adipose-derived stem cells; $\mathrm{CD}$, cluster of differentiation/surface antigen; $\mathrm{P}$, passage.

\section{Results}

Phenotypic characterization of ASCs by flow cytometry and changes over time with passaging. ASCs were harvested from inguinal fat pads of adult rats as plastic adherent cells through removal of nonadherent cells $24 \mathrm{~h}$ after cell harvesting and primary cell seeding. For characterization of the ASCs, cell surface protein expression was detected by flow cytometry (Fig. 1A). Through all three investigated passages, the majority of cells expressed the characteristic mesenchymal stem cell surface markers CD44 $(95.83 \pm 2.87 \%)$, CD73 $(83.92 \pm 9.23 \%)$, and CD90 $(98.23 \pm 1.61 \%)$, while $<5 \%$ of the cells were positive for CD11, CD105 and CD34 (Fig. 1B). Of note, the percentage of CD45-positive cells was $6.15 \pm 2.99 \%$ at passage 1 and increased significantly to $22.87 \pm 21.18 \%$ at passage 2 and $25.36 \pm 2.18 \%$ at passage 3 (Fig. 1B).

Differentiation capacity of ASCs. As mesenchymal stem cells are able to differentiate into adipogenic and osteogenic lineages, differentiation of isolated cell populations was induced into both directions. After 10 days in adipogenic differentiation medium, the existence of lipid vesicles could be verified by positive staining with Oil Red O (Fig. 2A). No staining was observed in the non-induced control samples (Fig. 2E). The osteogenic potential of ASCs was also evaluated. Four weeks after osteogenic induction, typical calcified nodules were identified, visualized by Alizarin red staining (Fig. 2B), in contrast to the non-induced ASC control population (Fig. 2F). Thirdly, chrondrogenic cells were identified by alcian blue staining in chondrogenicly-induced ASCs (Fig. 2C), while none were detected in the control non-induced samples (Fig. 2G).

To demonstrate neuronal differentiation capacity, ASCs were successfully differentiated into a SC-like phenotype. After 2 weeks of induction, cell shape alteration was observed from a flat, fibroblast-like structure into a spindle-shaped morphology, similar to SCs used as control (Fig. 2H). Successful differentiation to SC-like phenotype was further confirmed by detection of S100 expression (Fig. 2D). 

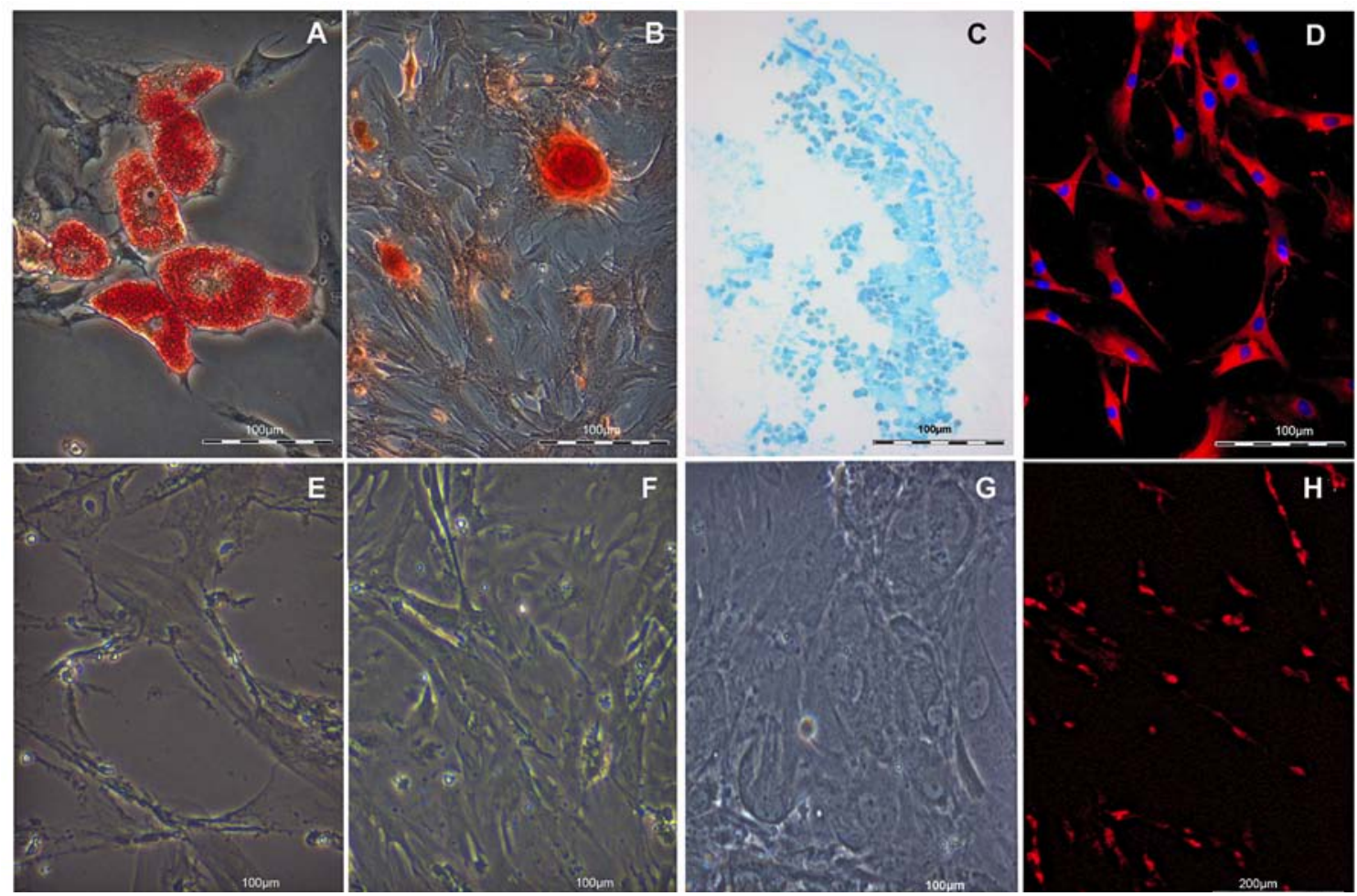

Figure 2. Induction of ASC differentiation as demonstration of pluripotency. The isolated ASCs underwent differentiation protocols to demonstrate their differentiation capacity. (A) Ten days after adipogenic differentiation lipid vesicles stained with oil red were detected. (B) Four weeks after osteogenic induction typical calcified nodules were observed, visualized by Alizarin red staining. (C) In osteogenic cultures, calcium depositions were confirmed by Alizarin red S staining. (D) SC phenotype was induced and subsequent morphology changes from fibroblastic flattened cells to a spindle shape cell type with positive immunostaining for the SC marker S100 could be observed. (E) Negative control for panel A. (F) Negative control for panel B. (G) Negative control for panel C. (H) SCs as a positive control for S100 staining. Scale bar, $100 \mu \mathrm{m}$. ASCs, adipose-derived stem cells; SCs, Schwann cells.
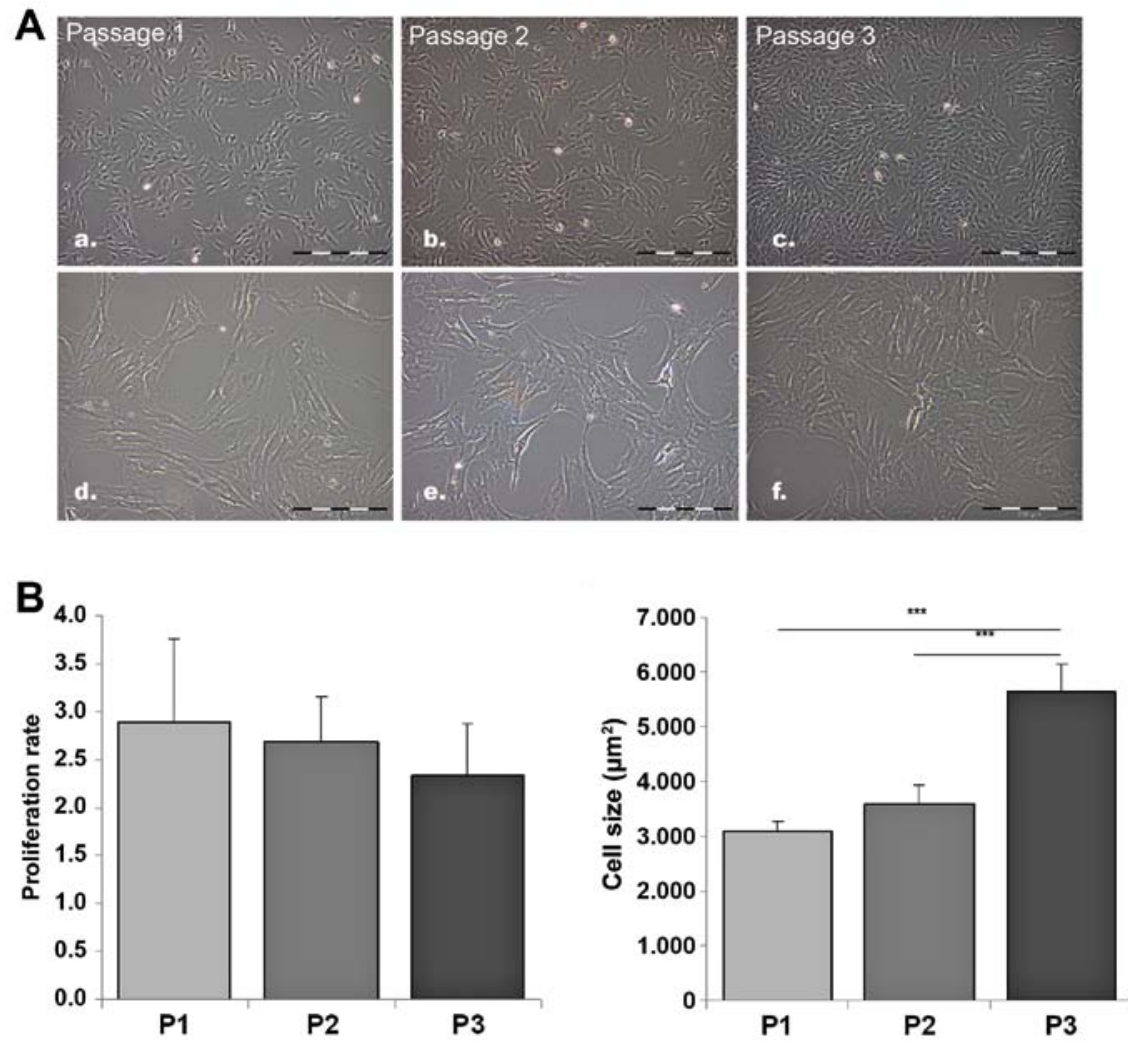

Figure 3. Morphologic characterization of ASCs and effect of cell passaging. (A) Representative microscopic images of the cell cultures. ASCs appeared as characteristic fibroblast-like cells in primary culture and were maintained in culture for up to 3 passages without significant changes in their fibroblast-like morphology. Panels a, b and c: scale bar, $500 \mu \mathrm{m}$; panels d, e and f, scale bar, $200 \mu \mathrm{m}$. (B) Cell proliferation and cell size were measured in ASCs of consecutive passages. Data are presented as means of 200 measured cells + standard deviation. ${ }^{* * *} \mathrm{P}<0.001$ with comparisons indicated by lines. ASCs, adipose-derived stem cells. 

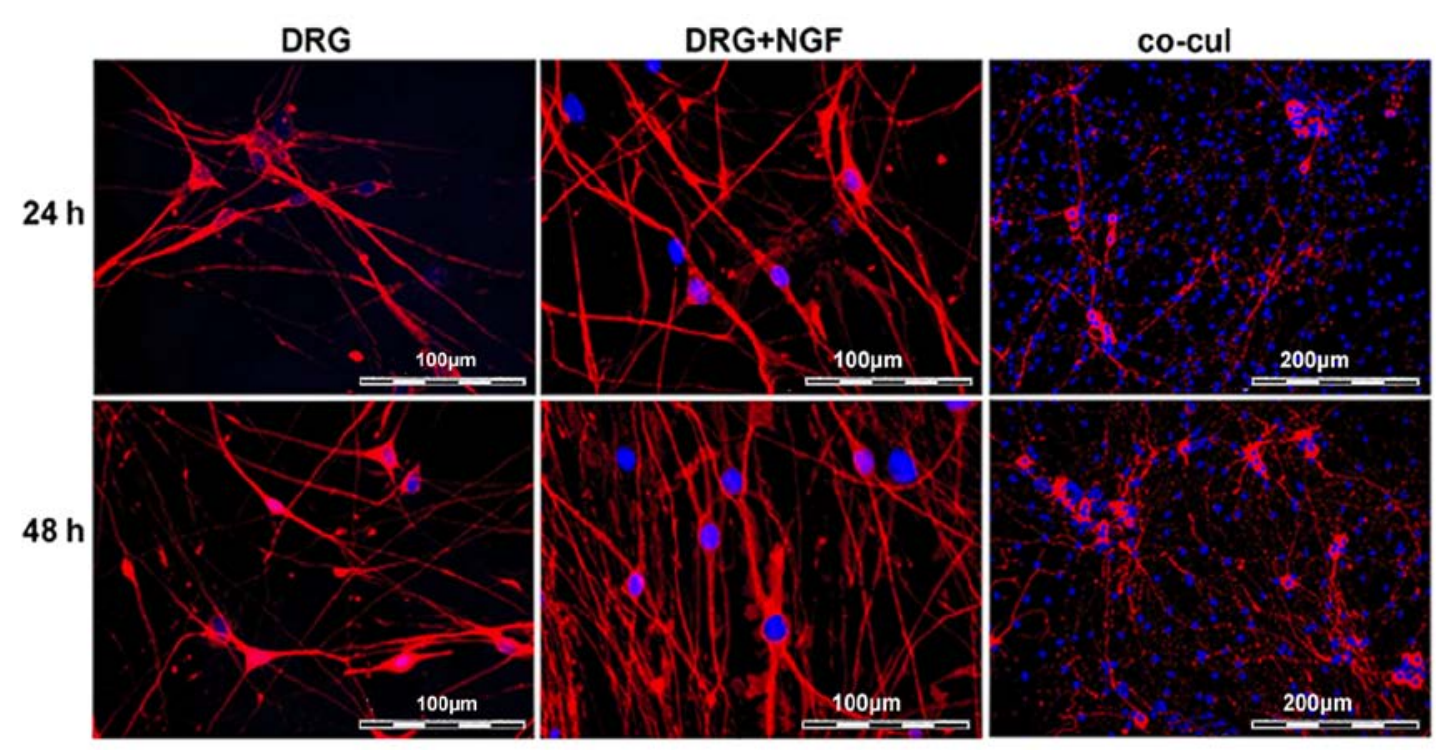

Figure 4. Microscopical analysis of neurons. Representative images from immunocytochemistry results for $\beta$-III-tubulin (red) staining in adult rat sensory neurons, as a marker of neurite outgrowth. The neurons were either cultivated in single cultures (DRG group), stimulated with NGF (NGF group), or co-cultured in direct contact with ASCs. Representative images from cultures at $24 \mathrm{~h}$ following seeding are presented in the upper row, while images from cultures at $48 \mathrm{~h}$ are presented in the lower row. DAPI (blue) was used to stain the nuclei. DRG, dorsal root ganglion; NGF, nerve growth factor; ASCs, adipose-derived stem cells.

Morphologic characterization of ASCs and effect of cell passaging. ASCs appeared as large and flat cells with a fibroblast-like, irregular shape. The cells continuously proliferated without significant changes in their fibroblast-like morphology until passage 3 (Fig. 3A). The proliferation rate was stable; the observed minimal decrease in proliferation rate with increasing passage numbers, from a 2.9 -fold at $24 \mathrm{~h}$ at passage 1 to a 2.3 -fold at $24 \mathrm{~h}$ at passage 3 , was not statistically significant (Fig. 3B). The cell size of subconfluent ASCs in progressive stages of cell culturing was increased by $82 \%$ in passage 3 compared with passage 1 (Fig. 3B).

Directed neurite outgrowth in co-culture with ASCs. Undifferentiated ASCs of passage 2 were co-cultured with freshly dissociated DRG neurons as an in vitro model of neurite outgrowth. Apart from the experimental group consisting of the co-cultured ASCs and DRG neurons, two control groups were used. The first contained neurons in single culture (DRG group) as a negative control, while the second contained neurons in DRG medium supplemented with NGF (NGF group) as a positive control. Under the culture conditions, neurites protruded from the seeded cells within $24 \mathrm{~h}$ in culture verified by immunostaining of $\beta$-III-tubulin (Fig. 4). After $48 \mathrm{~h}$, the number of neurites increased in all approaches, although the highest number of neurites was observed in the cultures supplemented with NGF (Fig. 4). In co-cultures with undifferentiated ASCs, an alignment of neurites along the ASCs could be observed, while ASCs themselves did not express $\beta$-III-tubulin (Fig. 4). To quantify the effect of ASCs on DRG neurons, several parameters were evaluated: percentage of neurons that formed neurites, number of neurites per neuron, mean and total length of neurites per neuron (Fig. 5A).

After 24 and $48 \mathrm{~h}$ of culture, the neurite outgrowth was compared between the three groups. Within few hours numerous neurons started to form neurites in all culture conditions. After 24 h, $46 \pm 16 \%$ of neurons in single culture and $56 \pm 13 \%$ of neurons in co-cultures exhibited neurites. At $48 \mathrm{~h}, 82 \pm 8 \%$ and $92 \pm 6 \%$, respectively, formed neurites.

Both after 24 and $48 \mathrm{~h}$, no significant difference was observed regarding the number of neurites per neuron formed in the three groups (Fig. 5A-a). ASC co-culture, however, appeared to have an influence on the neurite length at early time points. After $24 \mathrm{~h}$, the mean length of neurites was significantly increased in the co-cultures compared with the DRG group ( $\mathrm{P}=0.003$; Fig. 5A-b). After 48 h, a statistically significant difference between the three groups could not be detected any more (Fig. 5A-b). Similar results were observed when the whole length of neurites per neuron was analyzed (Fig. 5A-c).

According to the stimulating influence on neurite elongation, light microscopic analyses of the co-culture systems between ASCs and DRG neurons revealed a directed neurite elongation towards the ASCs. The neurites established direct contact with the ASC (Fig. 5B).

\section{Discussion}

The present study examined the feasibility of using ASCs as a source of stem cells for the differentiation of DRG neurons by an in vitro co-culture approach. Previous studies have demonstrated that rat ASCs can be differentiated toward a SC-like phenotype, which promotes neurite outgrowth and myelination $(33,34)$. In addition, ASCs secrete a plethora of growth factors that can mediate angiogenesis, wound healing, tissue regeneration and immune cell reactions $(35,36)$. In the regeneration of the nervous system, ASCs produce a wide variety of neurotrophic factors which can enhance neurite outgrowth and provide neuroprotection (37-40). It has been demonstrated that ASC-derived soluble factors in ASC-conditioned medium supported survival and proliferation of SCs and promoted neurite outgrowth in DRG neurons (41). However, the early effects of cell contact between undifferentiated ASC and DRG 
A

a

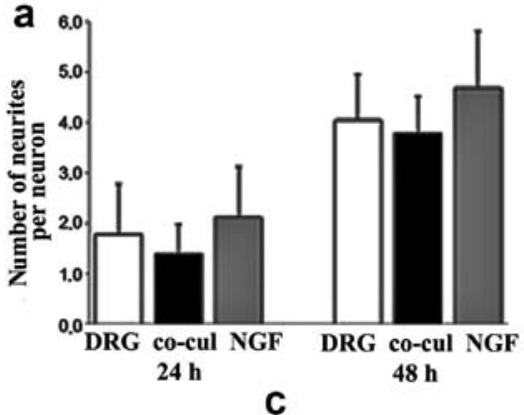

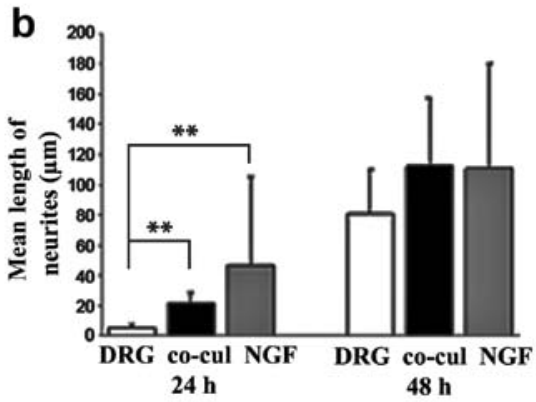

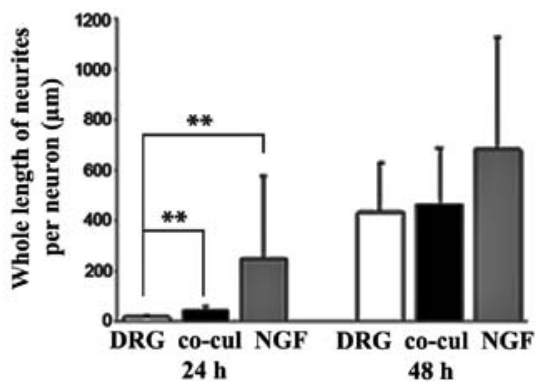

B

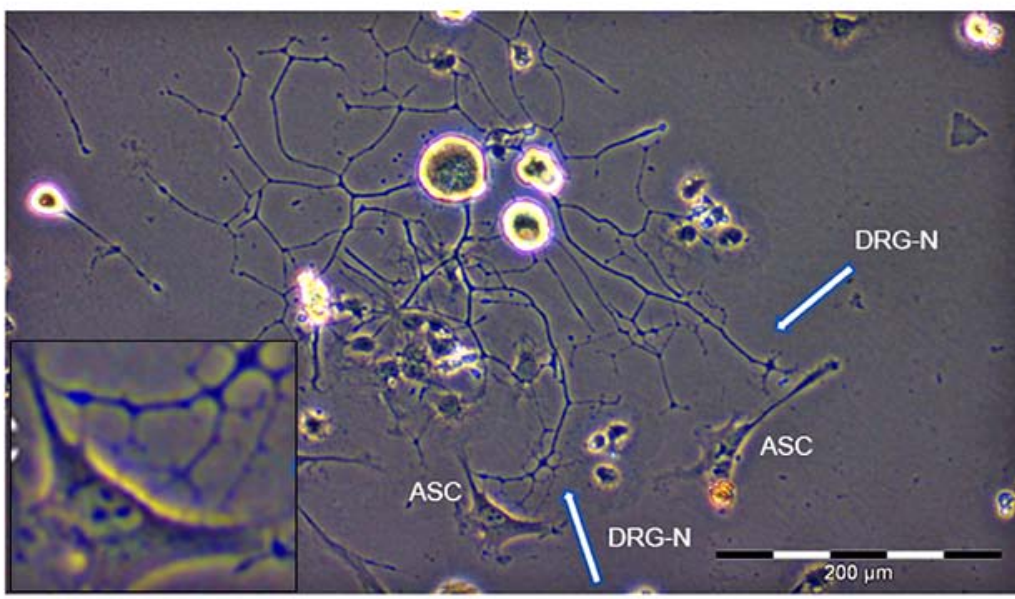

Figure 5. ASCs have a neurotrophic effect on DRG neurite outgrowth. (A) Morphometric analysis of co-cultures of ASCs with DRG neurons at 24 and $48 \mathrm{~h}$ following plating. The neurons from co-cultures of DRG neurons with ASC were compared with DRG neurons cultured alone in normal medium (DRG group) and with DRG neurons supplemented with NGF as positive control (NGF group). (a) Numbers of neurites per neuron, (b) mean and (c) total length of neurites per neuron were determined. Data are presented as means \pm standard deviation. ${ }^{* *} \mathrm{P}<0.01$ with comparisons indicated by lines. (B) Representative image from the co-culture of ASCs and DRG neurons at $48 \mathrm{~h}$ following plating. The image shows a neuron which has formed multiple, in part highly branched neurites and appears to contact the neighboring ASC. Scale bar, $200 \mu \mathrm{m}$. ASCs, adipose-derived stem cells; DRG, dorsal root ganglion; NGF, nerve growth factor; co-cul, co-culture.

neurons have not been investigated to date. The present study demonstrated that neurite outgrowth of DRG neurons was enhanced by co-culture with ASCs, as evidenced by increased neurite lengths even after short periods of co-culture at $24 \mathrm{~h}$. Acceleration of neurite length is of particular interest in peripheral nerve regeneration because the time period for successful regeneration is limited. Axonal regeneration and irreversible loss of neurovascular junction integrity are simultaneous ongoing processes, as long as the regenerating nerve has not reached its effector. Additionally, neurite branching was not increased in the present study, indicating that axonal sprouting is controlled by decreasing collateral axonal branching and possible reduced risk of neuroma formation (42).

The ASC populations were expanded over three passages without losing their proliferative capacity, which is in line with the findings of Mantovani et al (43), who described ASCs isolated from young adult rats with a significant reduction in their proliferation rate only starting with passage 20 . Nevertheless, there was a significant increase in cell size at passage 3, indicating cellular senescence (44). As an influence of replicative senescence on secretion of neurotrophic factors cannot be excluded (43), high passaging of ASCs was avoided in the present study and only cells from passage 2 were used for co-culture experiments.

The isolated ASCs were successfully characterized, as required by the International Society for Cell Therapy, for their expression of MSC-specific surface markers and their differentiation capacity into adipocytes, chondrocytes and osteoblasts. As expected, the primary cell cultures displayed a great percentage of CD44, CD73 and CD90-positive cells. 
High expression rates of CD44 have also been regarded as important for stemness of different adult stem cells (45), which might compensate for low levels of CD105 expression. The impact of CD105 expression on aspects of nerve regeneration is so far unknown and has to be characterized in further studies. In the present study, analysis of CD45 expression, a marker for hematopoietic lineage, revealed that its expression in the ASCs continuously increased from passage 1 to passage 3 . In the present study, analysis of CD45 expression, a marker for hematopoietic lineage, revealed that its expression in the ASCs continuously increased from passage 1 to passage 3. An increase in CD45 expression has also been described for late ASC passages in a previous study, which may indicate the ability of ASC long-term cultures to activate the immune response or an inclination towards hematopoietic differentiation (46). Proliferation rates of ASCs isolated from animals of different ages showed no significant difference and only small numbers of CD45-positive cells were contained in the cultures (41). The results of the present flow cytometry analyses confirmed the presence of MSC surface markers (CD73, CD90, CD44 >80\%; CD11, CD105, CD34<5\%; CD45 $>6 \%$ and $<25 \%$ ). Furthermore, the differentiation capacity of the isolated ASCs into adipocytes, chondrocytes and osteoblasts was confirmed. The present panel of surface markers does not fully meet the criteria introduced by Dominici et al (47). However, based on the present surface phenotype analysis, in conjunction with the differentiation functional criteria, the isolated ASC cultures were confirmed based on the current state of knowledge. Strict observation of the cultures concerning their proper phenotype, differentiation and senescence is an important factor for optimized results concerning stimulation of neurite growth. These findings were taken into account in the experimental design of the present study, avoiding high passages of the cells. These strict criteria will also be important for future studies in vivo.

Furthermore, the ASCs in passage 2 could successfully be induced for chondrogenic, osteogenic, adipose and glial cell lineage differentiation. Additionally, it was demonstrated that ASCs could be differentiated into a functional SC-like phenotype, expressing markers S100 and enhancing neurite outgrowth in vitro. The ASC neurotrophic potential has also been confirmed by in vivo peripheral nerve repair studies $(48,49)$, indicating that adult stem cells may be of benefit for treatment of peripheral nerve injuries.

A direct contact of severed nerves with growth factors, such as NGF, can lead to extreme pain and hyperexcitability (6). Therefore, the use of ASCs as a source of growth factors in the clinic may be advantageous, because an uncontrollable growth factor supplementation might lead to severe side effects. This is in contrast to differentiated ASCs where secretion in high amounts of neurothrophins and growth factors was demonstrated (41). This does not exclude the ability of undifferentitated ASCs to produce growth factors, but their production and secretion of growth factors might be more targeted than an unspecific, uncontrollable exogenous supplementation. As cell-to-cell contact in co-culture with direct neuritic contact on the cell surface of the undifferentiated ASCs was observed in the present study, the positive neuronal influence might be based on other cell signaling mechanisms or direct crosstalk between the cells.
In the present study, undifferentiated ASCs were used due to their advantages in regard to possible clinical applications. The majority of studies have demonstrated the efficacy of ASCs following glial differentiation (50). In the case of a potential clinical transfer, the application of unaltered cells is preferable. This underlines the need for a more detailed knowledge regarding the interaction and efficacy of undifferentiated ASCs on injured neurons, in order to optimize the healing results and to reduce undesired side effects.

The present results demonstrated that undifferentiated ASCs significantly enhanced the speed of DRG neuron neurite growth in a co-culture system. This effect is important for nerve injury treatment, as a quick growth of neurites is crucial for success in peripheral nerve regeneration. These findings indicate that undifferentiated ASCs may have promise as a method of direct transplantation in peripheral nerve regeneration.

\section{Acknowledgements}

The authors would like to thank Miss Jieli Liu for helping with the measurements of the neuronal parameters. The authors would also thank Dr Stefanie Michael for writing assistance.

\section{Funding}

The present study has been supported by the Boehringer Ingelheim Foundation (grant no. 19470076).

\section{Availability of data and materials}

The analyzed datasets generated during the present study are available from the corresponding author on reasonable request.

\section{Authors' contributions}

RS, SS and AF performed experiments and analyzed the data. VB, DV and CTP analyzed the data and participated in the interpretation of the data. PMV, KR and MF participated in the interpretation of the data. KR, SS and MF revised the manuscript critically. CR designed, analyzed and interpreted the study. VB prepared the manuscript. All authors read and approved the final manuscript.

\section{Ethics approval and consent to participate}

All animals were treated according to the legal and ethical requirements of the German Animal Welfare Act and were approved by the Animal Ethics Committee of the Hannover Medical School Central Animal Laboratory (approval no. 2014/52).

\section{Patient consent for publication}

Not applicable.

\section{Competing interests}

The authors declare that they have no competing interests. 


\section{References}

1. Madduri S and Gander B: Schwann cell delivery of neurotrophic factors for peripheral nerve regeneration. J Peripher Nerv Syst 15: 93-103, 2010.

2. Chimutengwende-Gordon M and Khan W: Recent advances and developments in neural repair and regeneration for hand surgery. Open Orthop J 6: 103-107, 2012.

3. Reid AJ, Sun M, Wiberg M, Downes S, TerenghiG and Kingham PJ: Nerve repair with adipose-derived stem cells protects dorsal root ganglia neurons from apoptosis. Neuroscience 199: 515-522, 2011

4. Wong FS, Chan BP and Lo AC: Carriers in cell-based therapies for neurological disorders. Int J Mol Sci 15: 10669-10723, 2014.

5. Ide C: Peripheral nerve regeneration. Neurosci Res 25: 101-121, 1996.

6. Radtke C, Wewetzer K, Reimers K and Vogt PM: Transplantation of olfactory ensheathing cells as adjunct cell therapy for peripheral nerve injury. Cell Transplant 20: 145-152, 2011.

7. Tohill M and Terenghi G: Stem-cell plasticity and therapy for injuries of the peripheral nervous system. Biotechnol Appl Biochem 40: 17-24, 2004

8. Muschler GF, Nitto H, Boehm CA and Easley KA: Age- and gender-related changes in the cellularity of human bone marrow and the prevalence of osteoblastic progenitors. J Orthop Res 19 $117-125,2001$.

9. Strem BM, Hicok KC, Zhu M, Wulur I, Alfonso Z, Schreiber RE, Fraser JK and Hedrick MH: Multipotential differentiation of adipose tissue-derived stem cells. Keio J Med 54: 132-141, 2005.

10. Robinton DA and Daley GQ: The promise of induced pluripotent stem cells in research and therapy. Nature 481: 295-305, 2012.

11. Walsh S and Midha R: Use of stem cells to augment nerve injury repair. Neurosurgery 65 (Suppl): A80-A86, 2009.

12. Weinstein DE: The role of Schwann cells in neural regeneration. Neuroscientist 5: 208-216, 1999.

13. Ikeda M, Uemura T, Takamatsu K, Okada M, Kazuki K, Tabata Y, Ikada Y and Nakamura H: Acceleration of peripheral nerve regeneration using nerve conduits in combination with induced pluripotent stem cell technology and a basic fibroblast growth factor drug delivery system. J Biomed Mater Res A 102: 1370-1378, 2014

14. Satarian L, Javan M, Kiani S, Hajikaram M, Mirnajafi-Zadeh J and Baharvand $\mathrm{H}$ : Engrafted human induced pluripotent stem cell-derived anterior specified neural progenitors protect the rat crushed optic nerve. PLoS One 8: e71855, 2013.

15. Uemura T, Takamatsu K, Ikeda M, Okada M, Kazuki K, Ikada Y and Nakamura H: Transplantation of induced pluripotent stem cell-derived neurospheres for peripheral nerve repair. Biochem Biophys Res Commun 419: 130-135, 2012.

16. Wang A, Tang Z, Park IH, Zhu Y, Patel S, Daley GQ and Li S: Induced pluripotent stem cells for neural tissue engineering. Biomaterials 32: 5023-5032, 2011

17. Rodriguez AM, Elabd C, Amri EZ, Ailhaud G and Dani C: The human adipose tissue is a source of multipotent stem cells. Biochimie 87: 125-128, 2005.

18. Gimble JM, Katz AJ and Bunnell BA: Adipose-derived stem cells for regenerative medicine. Circ Res 100: 1249-1260, 2007.

19. Carvalho PP, Wu X, Yu G, Dias IR, Gomes ME, Reis RL and Gimble JM: The effect of storage time on adipose-derived stem cell recovery from human lipoaspirates. Cells Tissues Organs 194: 494-500, 2011.

20. Radtke C, Schmitz B, Spies M, Kocsis JD and Vogt PM: Peripheral glial cell differentiation from neurospheres derived from adipose mesenchymal stem cells. Int J Dev Neurosci 27: 817-823, 2009.

21. Ren Z, Wang Y, Peng J, Zhao Q and Lu S: Role of stem cells in the regeneration and repair of peripheral nerves. Rev Neurosci 23: 135-143, 2012

22. Papalia I, Raimondo S, Ronchi G, Magaudda L, GiacobiniRobecchi MG and Geuna S: Repairing nerve gaps by vein conduits filled with lipoaspirate-derived entire adipose tissue hinders nerve regeneration. Ann Anat 195: 225-230, 2013.

23. Georgiou M, Golding JP, Loughlin AJ, Kingham PJ and Phillips JB: Engineered neural tissue with aligned, differentiated adipose-derived stem cells promotes peripheral nerve regeneration across a critical sized defect in rat sciatic nerve. Biomaterials 37: 242-251, 2015.

24. Hsueh YY, Chang YJ, Huang TC, Fan SC, Wang DH, Chen JJ, $\mathrm{Wu} C \mathrm{C}$ and Lin SC: Functional recoveries of sciatic nerve regeneration by combining chitosan-coated conduit and neurosphere cells induced from adipose-derived stem cells. Biomaterials 35: 2234-2244, 2014.
25. Orbay H, Uysal AC, Hyakusoku H and Mizuno H: Differentiated and undifferentiated adipose-derived stem cells improve function in rats with peripheral nerve gaps. J Plast Reconstr Aesthet Surg 65: 657-664, 2012

26. Sun F,Zhou K, Mi WJ and Qiu JH: Combined use of decellularized allogeneic artery conduits with autologous transdifferentiated adipose-derived stem cells for facial nerve regeneration in rats. Biomaterials 32: 8118-8128, 2011.

27. Ravasi M, Scuteri A, Pasini S, Bossi M, Menendez VR, Maggioni D and Tredici G: Undifferentiated MSCs are able to myelinate DRG neuron processes through p75. Exp Cell Res 319: 2989-2999, 2013.

28. Razavi S, Mardani M, Kazemi M, Esfandiari E, Narimani M, Esmaeili A and Ahmadi N: Effect of leukemia inhibitory factor on the myelinogenic ability of Schwann-like cells induced from human adipose-derived stem cells. Cell Mol Neurobiol 33: 283-289, 2013.

29. Erba P, Mantovani C, Kalbermatten DF, Pierer G, Terenghi G and Kingham PJ: Regeneration potential and survival of transplanted undifferentiated adipose tissue-derived stem cells in peripheral nerve conduits. J Plast Reconstr Aesthet Surg 63: e811-e817, 2010.

30. Faroni A, Smith RJ and Reid AJ: Adipose derived stem cells and nerve regeneration. Neural Regen Res 9: 1341-1346, 2014.

31. Kolar MK and Kingham PJ: Regenerative effects of adiposetissue-derived stem cells for treatment of peripheral nerve injuries. Biochem Soc Trans 42: 697-701, 2014.

32. Kingham PJ, Kalbermatten DF, Mahay D, Armstrong SJ, Wiberg $\mathrm{M}$ and Terenghi G: Adipose-derived stem cells differentiate into a Schwann cell phenotype and promote neurite outgrowth in vitro. Exp Neurol 207: 267-274, 2007.

33. di Summa PG, Kalbermatten DF, Raffoul W, Terenghi G and Kingham PJ: Extracellular matrix molecules enhance the neurotrophic effect of Schwann cell-like differentiated adipose-derived stem cells and increase cell survival under stress conditions. Tissue Eng Part A 19: 368-379, 2013.

34. Xu Y, Liu L, Li Y, Zhou C, Xiong F, Liu Z, Gu R, Hou X and Zhang C: Myelin-forming ability of Schwann cell-like cells induced from rat adipose-derived stem cells in vitro. Brain Res 1239: 49-55, 2008.

35. Kapur SK and Katz AJ: Review of the adipose derived stem cell secretome. Biochimie 95: 2222-2228, 2013

36. Salgado AJ, Reis RL, Sousa NJ and Gimble JM: Adipose tissue derived stem cells secretome: Soluble factors and their roles in regenerative medicine. Curr Stem Cell Res Ther 5: 103-110, 2010.

37. Chung JY, Kim W, Im W, Yoo DY, Choi JH, Hwang IK, Won MH, Chang IB, Cho BM, Hwang HS, et al: Neuroprotective effects of adipose-derived stem cells against ischemic neuronal damage in the rabbit spinal cord. J Neurol Sci 317: 40-46, 2012.

38. Kalbermatten DF, Schaakxs D, Kingham PJ and Wiberg M: Neurotrophic activity of human adipose stem cells isolated from deep and superficial layers of abdominal fat. Cell Tissue Res 344: 251-260, 2011.

39. Lattanzi W, Geloso MC, Saulnier N, Giannetti S, Puglisi MA, Corvino V, Gasbarrini A and Michetti F: Neurotrophic features of human adipose tissue-derived stromal cells: In vitro and in vivo studies. J Biomed Biotechnol 2011: 468705, 2011.

40. Wei X, Zhao L, Zhong J, Gu H, Feng D, Johnstone BH, March KL, Farlow MR and Du Y: Adipose stromal cells-secreted neuroprotective media against neuronal apoptosis. Neurosci Lett 462: 76-79, 2009.

41. Sowa Y, Imura T, Numajiri T, Nishino K and Fushiki S: Adiposederived stem cells produce factors enhancing peripheral nerve regeneration: Influence of age and anatomic site of origin. Stem Cells Dev 21: 1852-1862, 2012.

42. Skouras E, Ozsoy U, Sarikcioglu L and Angelov DN: Intrinsic and therapeutic factors determining the recovery of motor function after peripheral nerve transection. Ann Anat 193: 286-303, 2011.

43. Mantovani C, Raimondo S, Haneef MS, Geuna S, Terenghi G Shawcross SG and Wiberg M: Morphological, molecular and functional differences of adult bone marrow- and adipose-derived stem cells isolated from rats of different ages. Exp Cell Res 318: 2034-2048, 2012.

44. Simons JW: The use of frequency distributions of cell diameters to characterize cell populations in tissue culture. Exp Cell Res 45: 336-350, 1967.

45. Maleki M, Ghanbarvand F, Reza Behvarz M, Ejtemaei M and Ghadirkhomi E: Comparison of mesenchymal stem cell markers in multiple human adult stem cells. Int J Stem Cells 7: 118-126, 2014.

46. Wan Safwani WK, Makpol S, Sathapan S and Chua KH: The changes of stemness biomarkers expression in human adiposederived stem cells during long-term manipulation. Biotechnol Appl Biochem 58: 261-270, 2011. 
47. Dominici M, Le Blanc K, Mueller I, Slaper-Cortenbach I, Marini F, Krause D, Deans R, Keating A, Prockop Dj and Horwitz E: Minimal criteria for defining multipotent mesenchymal stromal cells. The International Society for Cellular Therapy position statement. Cytotherapy 8: 315-317, 2006.

48. di Summa PG, Kalbermatten DF, Pralong E, Raffoul W, Kingham PJ and Terenghi G: Long-term in vivo regeneration of peripheral nerves through bioengineered nerve grafts. Neuroscience 181: 278-291, 2011.
49. di Summa PG, Kingham PJ, Raffoul W, Wiberg M, Terenghi G and Kalbermatten DF: Adipose-derived stem cells enhance peripheral nerve regeneration. J Plast Reconstr Aesthet Surg 63: 1544-1552, 2010.

50. Zuck P: Adipose-derived stem cells in tissue regeneration. ISRN Stem Cells 2013: e713959, 2013. 